\title{
Structure of Patt1 human proapoptotic histone acetyltransferase
}

\author{
Roch Pawel Jẹdrzejewski • Rajmund Kaźmierkiewicz
}

Received: 27 July 2013 / Accepted: 21 October 2013 /Published online: 19 November 2013

(C) The Author(s) 2013. This article is published with open access at Springerlink.com

\begin{abstract}
The results of modeling of a novel human histone acetyltransferase Patt1 are presented here. This protein belongs to the GNAT GCN5 family and shows proapoptotic activity in human hepatocellular carcinoma cells. Patt1 is an attractive therapeutic target. The sequence analysis, fold recognition predictions and homology modeling of Patt1 protein structure were performed. N- and C- termini of Patt1 were unstructured. Central part revealed classical GNAT fold-central 7 -stranded beta sheet core surrounded by intervening 4 alpha helices. The model was assessed with the methods for protein structure validation PROQ and MetaMQAPII. The allatom $12 \mathrm{~ns}$ molecular dynamics simulation of Patt1 model with TIP3P water model and counterions was conducted. All assessment methods implemented resulted in conviction that the model was of quality that could provide confident structural information to infer sequence-structure-function relationships of Patt1. Phe186 and Cys137 were identified as residues engaged in acetyltransfer reaction and the clues for the identification of reaction mechanism were proposed. The knowledge of detailed molecular architecture of Patt1 is not only the key to understanding its mechanistic functional properties but it also opens the possibility of rational drug and protein design experiments, leading to development of effective therapeutic methods.
\end{abstract}

Keywords GNAT family proteins - HAT histone acetyltransferases · Homology modeling · Molecular dynamics simulation . Protein structure prediction

R. P. Jędrzejewski $(\bowtie) \cdot$ R. Kaźmierkiewicz Laboratory of Biomolecular Systems Simulations, Intercollegiate Faculty of Biotechnology, University of Gdańsk and Medical University of Gdańsk, Kładki 24, 80-822 Gdańsk, Poland e-mail: rochjedrzejewski@gmail.com

\section{Introduction}

In eukaryotic cells the acetylation of histone $\mathrm{N}$-terminal tails is the key regulatory mechanism in histone code and the execution of epigenetic information [1-3]. The information encoded in modification patterns of $\mathrm{N}$-terminal histone tails is crucial for chromatin remodeling. The modification of tails is then read out by specific proteins by means of molecular recognition. Chromatin remodeling machines (CRM) complexes take the input information encoded in the form of the modifications order. This drives the chromatin remodeling processes and opens access to chromatin. These events transfer chromatin to transcriptionally active state in which DNA template is accessible for transcription factors and other regulatory proteins that bind DNA. This results in expression of specific genes. Switching chromatin to an open state is induced by acetylation [1-3]. Acetylation is performed by vast array of enzymes from various families [4]. Many different families of proteins are engaged in histone acetylation $[5,6]$. Histone acetyltransferases (HAT) perform acetylation of N-terminal histone tails at specific lysines. Dysfunction of histone acetyltransferases leads to carcinogenic processes [7, 8]. This aspect of acetyltransferases has gained growing attention in recent years. The GNAT family of acetyltransferases [9-11] contains the first discovered proteins that possess histone acetylation activity that was connected with gene activation [12]. A recently discovered member of this group Patt 1 (protein acetyltransferase-1) has been shown to acetylate histone H4 in vitro and in vivo [13]. Activity of this protein was linked to promotion of apoptosis in human hepatocellular carcinoma cells. There is no information available about the structure of this new human histone acetyltransferase. Pattl protein allatom tertiary structure was modeled with the use of theoretical methods. All structure assessment methods and the results of molecular dynamics simulation indicated that the model is correct. The model exhibits all features of GNAT canonical 
topology: the core of protein is formed by central beta sheet composed of seven strands. Alpha helices are mixed with beta sheets and surround the structure on both sides. The enzyme active site is located at the edge of beta sheet, between $\beta 5$ and $\beta 6$ structural elements. Amino acid residues engaged in acetyltransfer reaction were identified: the aromatic rings of Phe185, Phe186, and Phe192 form a stacking system. Their role is alternative to general acid catalysis. The spatial localization of Cys137 is similar to analogous cysteine residue in Esa1 histone acetyltransferase where acetyltransfer reaction proceeds through an acetyl-cysteine enzyme intermediate. The model represents the first structural data regarding Patt1 protein.

\section{Materials and methods}

Patt1 sequence analysis

The searches of an nr National Center for Biotechnology Information (NCBI) sequence database [14] were performed using a PSI-BLAST algorithm [15] with Patt1 sequence [13] as an input (query). Default parameters were used, except for the "max target sequences" which was set to 1000 . After eight iterations the set of resulting sequences showing statistically significant similarity was collected and used to construct the multiple sequence alignment (MSA) of Patt1 family. MSA was computed with the use of MUSCLE program [16, 17]. CDS (conserved domain search) [18] was used for assignment of the sequence to specific protein superfamily. Manipulation of sequence data was done with use of BioEdit [19] and SeaView [20] programs. ClustalX [21] was used to build phylogenetic trees (data not shown). TreeView [22] program was used to visualize them.

\section{Fold recognition analysis}

Fold recognition methods are based on detection of compatibility of a given protein sequence with sequence profiles and/ or structures of proteins deposited in structural databases. In order to identify a suitable template for homology modeling of Patt1 structure the MSA of Patt1 family was submitted to GeneSilico Metaserver [23], which is a gateway to $>30$ third party methods of automated protein secondary structure prediction, solvation and disorder prediction (reference: https:// genesilico.pl/meta2/).

\section{Patt1 structure modeling}

The MODELLER program [24] available through GeneSilico Web Toolkit (https://genesilico.pl/toolkit/modeling) was used to construct an all-atom tertiary model of Patt1. Swiss-Pdb
Viewer (SPDBV) program [25] was used for structure visualization and manipulation.

Model quality assessment

All theoretical models must be thoroughly evaluated before using them as source of information of molecular architecture and composition of key structural elements. The state-of-theart methods of protein structure quality assessment specially designed to score theoretical models were used. Among them were MetaMQAPII [26] and ProQ [27] methods implemented in GeneSilico Web Toolkit (https://genesilico.pl/toolkit).

The ProQ web server [28] (available at Stockholm Bioinformatics Center website: http://www.sbc.su.se/ bjornw/ ProQ/ProQ.html) was also used.

Energy minimization and molecular dynamics simulations

Molecular dynamics simulation and energy minimization were carried out using AMBER9 package [29]. Initial energy minimization in vacuo with unrestrained system was performed. Steepest descent algorithm was used for the first 6000 steps and conjugate gradient algorithm for following 24,000 steps. Cutoff value for treating long-range electrostatic interactions was 12.0. Simulation was performed with explicit solvent water model TIP3P. Counterions were added to neutralize net charge on protein surface. Long-range electrostatic interactions were evaluated using particle mesh Ewald summation method with periodic boundary conditions. Langevin thermostat method was used to control temperature of the system. The temperature was set to $300 \mathrm{~K}$. The simulation duration was $12 \mathrm{~ns}$, with time step (dt) of $2 \mathrm{fs}$.

\section{Results and discussion}

Patt1 sequence analysis

The sequence of Patt1 protein is 237 amino acid residues long. The set of sequences resulting from preliminary PSI-BLAST (default parameters, except for the "max target sequences = 1000 " setting) search contained various members of GNAT family acetyltransferases from all domains of life. According to CDS results the region of similarity to GNAT superfamily was detected between approximately $112-187$ amino acid residues. MSA was constructed and submitted to GeneSilico Metaserver. Disorder prediction indicated that the N-terminal (1-50 amino acid residues) and C-terminal regions (215-237 amino acid residues) of Patt1 protein were unstructured. The predicted regions of alpha helices and beta sheets conformed to the $(\alpha)-\beta-\alpha-\alpha-\beta-\beta-\beta-\alpha-\beta-\alpha-\beta-\beta$ pattern, which is in agreement with the canonical GNAT fold topology (Fig. 1). The prediction of first alpha helix is uncertain since it occurs 


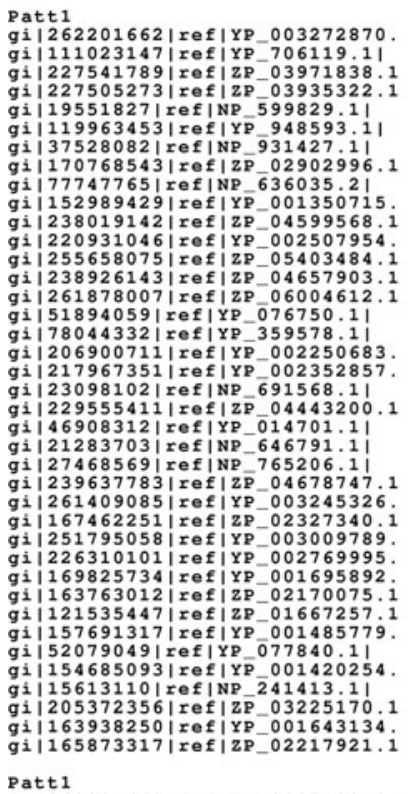

Patt 1

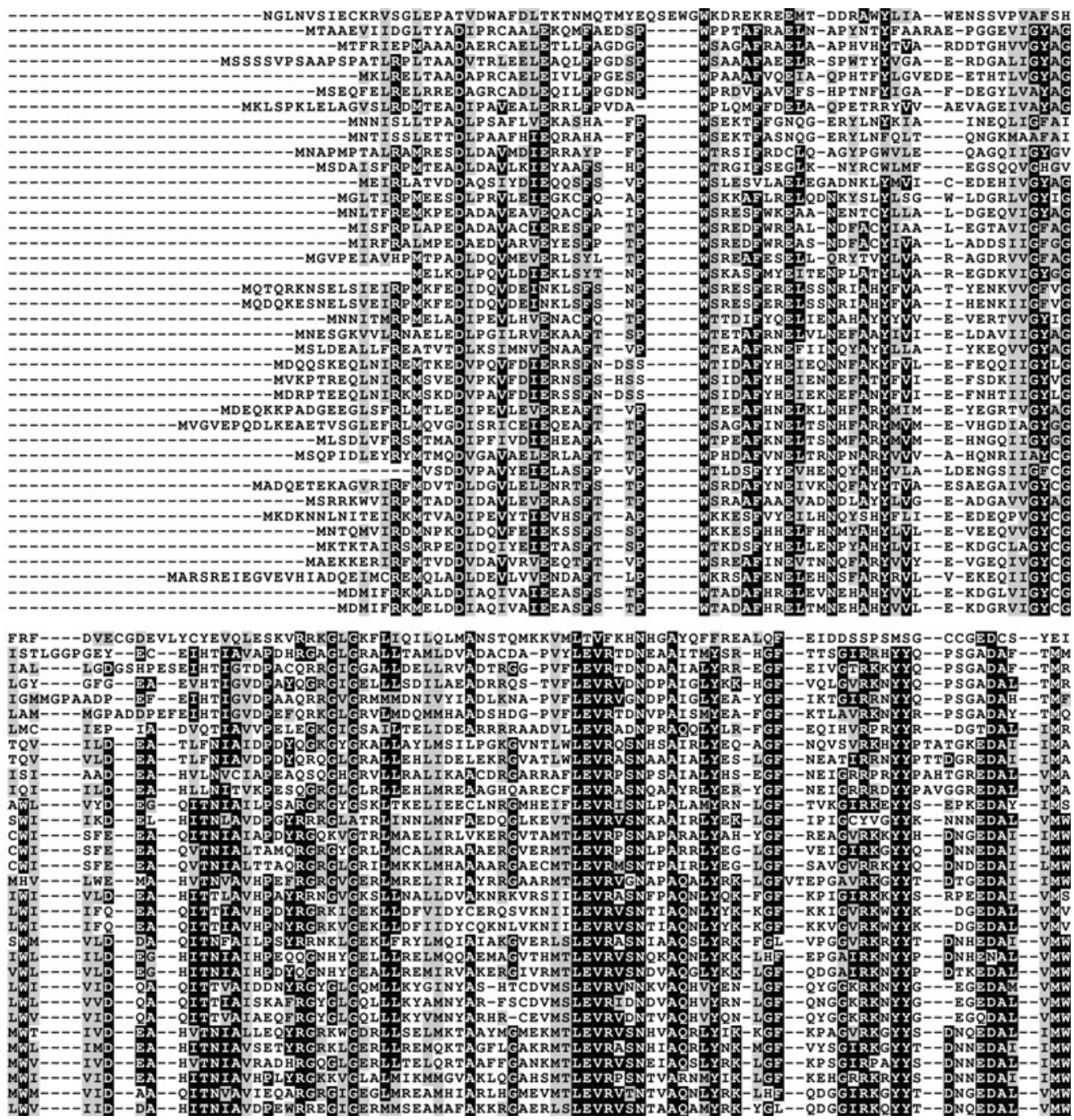
i) 227541789 I gi|227505273|ref|zP 03935322 . gi|19551827|ref|NP_59989.1| gi| 37528082 |ref|NP 931427 . i gi| $170768543 \mid$ ref|ZP 02902996 gi 152989429 ref Y $\bar{p} 0013507$ i) 238019142 | ref|zP 04599568 . gi $|220931046|$ ref |YP 002507954 gi|255658075|ref|zP_05403484. (1) gi|261878007|ref|zP_06004612 gi| 51894059 |ref|YP_076750.1 i | 206900711 |ref|YP_002250683. gi 217967351 |ref|YP 002352857 . gi $|23098102| \mathrm{ref} \mid \mathrm{NP}-691568$.1 Ii 22955411 ref|zP_-04443200. gi 469312 ref YP_014701.1 gi| $27468569 \mid$ ref|NP_765206.1 1 39637783 |ref|ZP_04678747. $85|r e f| Y P+003245326$ i) 251795058 |ref|YP gi|226310101|ref|YP-0027699 gi|169825734|ref|YP-001695892

Fig. 1 Multiple sequence alignment of Patt1 amino acid sequence (residues 50-237) and representative sequences of significant similarity to Patt1. GNAT family conserved sequence motifs [11] are discernible

in the region of predicted disorder and is likely to represent "intrinsically disordered" locally stable alpha helix in the lack of defined tertiary structure.

Fold recognition and Patt1 structure modeling

Fold recognition methods implemented in GeneSilico Metaserver were used to search within PDB database for protein structures solved by X-ray crystallography and NMR. The structure scoring by fold recognition servers provides means of selection of suitable template structures. Fold recognition servers returned many structures that can be used as possible templates in homology modeling. The templates were in most cases members of a newly discovered GNAT subfamily of pita-like proteins. The choice was based on sequence similarity and structure scoring by fold recognition servers. The structure of RimI [30] was chosen as a modeling template. The Patt1 model was constructed with use of the MODELLER program.

Patt1 model assessment

The Pattl model was scored by ProQ and MetaMQAPII methods. The ProQ LGscore: 2.082 and MaxSub: 0.275 indicated that the model was of sufficient quality "fairly good model", and can be used in subsequent stages of analysis. MetaMQAPII confirmed correctness of the model.

Patt1 molecular dynamics

The binding site of the peptide and $\mathrm{AcCoA}$ is located at the catalytic center of the enzyme, surrounded by B-sheet elements $\beta 6$ and $\beta 7$. The extended loop between these structural elements showed highest conformational flexibility during 

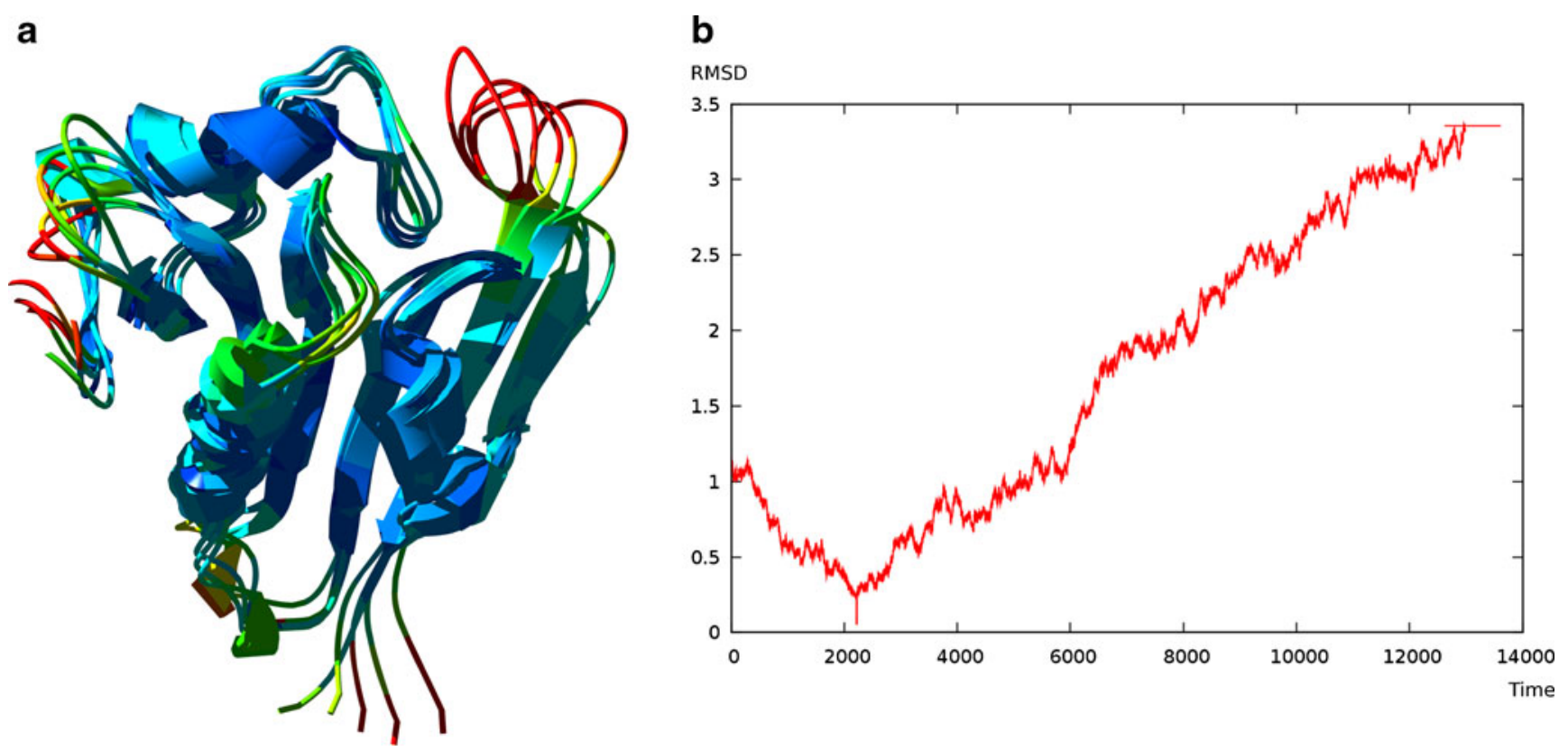

Fig. 2 a Ensemble of structures from MD simulation representing stages of the model after 4, 6, 8, 10 and 12 ns. Colors corresponds to RMSD values. b Plot of RMSD value averaged for $\mathrm{C}$-alpha atoms over time

simulation. This behavior corresponds with available literature data for RimI where $\beta 6-\beta 7$ loop movements correlate with acetyltransfer reaction. The protein structure was stable during simulation, no unfolding of tertiary structure was observed (Fig. 2).

\section{Patt1 structure analysis}

The region that comprises GNAT fold domain is located between 59 and 220 amino acid residue. Patt1 reveals classical
GNAT fold with the central region of beta sheet surrounded by alpha helices. The sequence of Patt1 structural elements is: $\beta 1-\alpha 1-\alpha 2-\beta 2-\beta 3-\beta 4-\alpha 3-\beta 5-\alpha 4-\beta 6-\beta 7$ (Fig. 3). In the core region, the enzyme active site is located at the edge of the central beta sheet, surrounded by $\alpha 3$ and $\alpha 4$ helices in the region of Acetyl-CoA binding. The $\beta 6-\beta 7$ sheet structural elements along with the loop between $\alpha 1$ and $\alpha 2$ helices enclose the active site in the region of acetylated peptide binding. The characteristic feature of GNAT family proteins, the V-like splay shape between the $\beta 4-\beta 5$ (the specific region

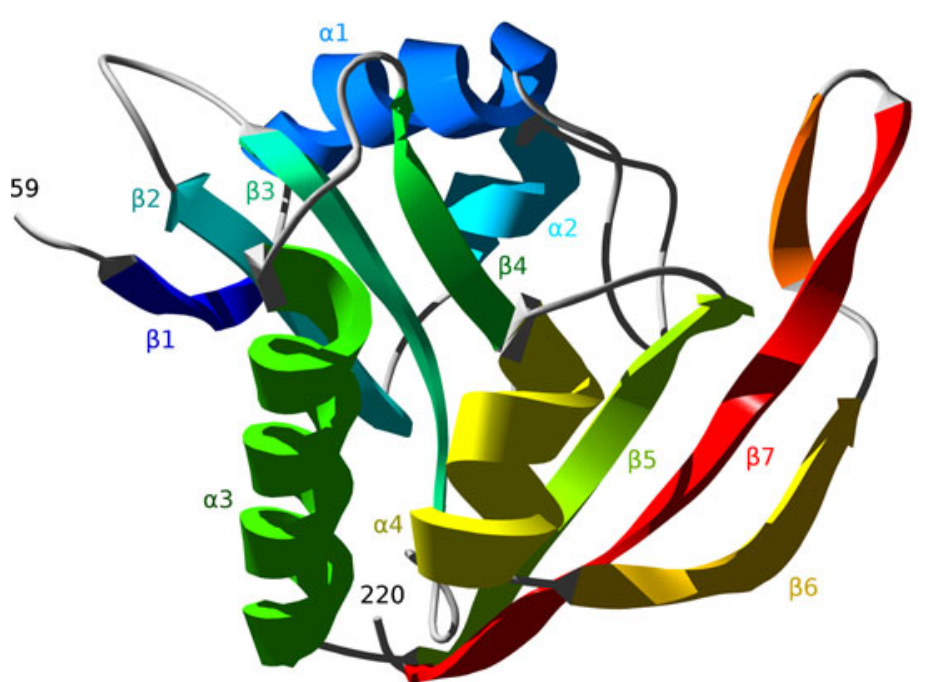

Fig. 3 Overview of the Patt1 structure. Schematic ribbon representation featuring sheets and helices. The second picture is rotated by $90^{\circ}$ along $\mathrm{x}$ axis into image plane, giving impression of "top

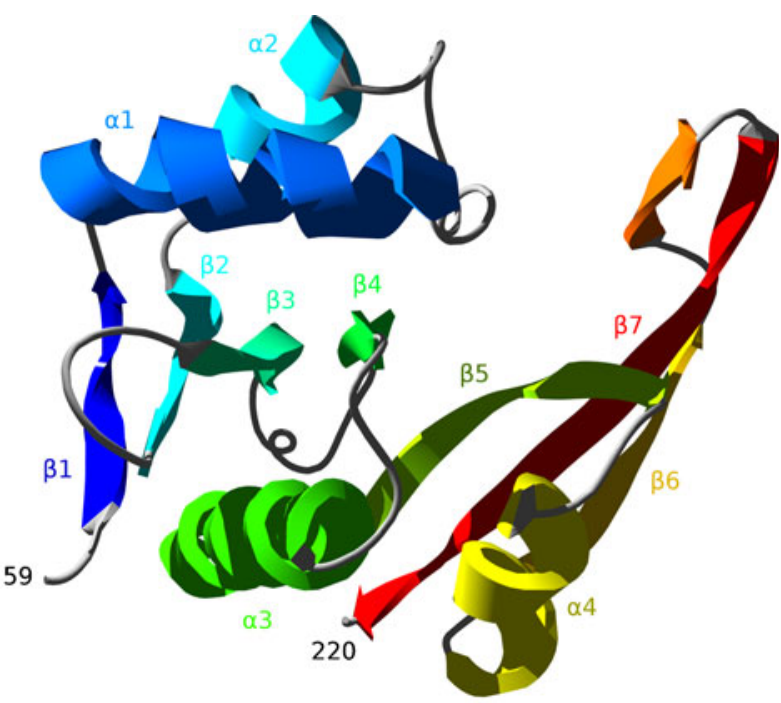

view" over the edge of the central $\beta$-sheet. The $\beta$-like structural element located between $\beta 6-\beta 7$ sheets is unstable and dissolves during MD simulation into extended loop 
of active site where acetyltransfer reaction takes place) structural elements is also present in the Pattl model. The high quality of the model allows detailed predictions about the specific amino acid residues function to be made. The sequence region "RRKGLG" spanning between 147 and 152 amino acid residues in Pattl sequence was shown to be the GNAT signature motif Arg/Gln-X-X-Gly-X-Gly/Ala responsible for recognition and binding of $\mathrm{CoA}$ [13]. In the model it is located in the loop between $\beta 4-\alpha 3$ and at the beginning of $\alpha 3$ helix. Particularly interesting are other interactions with $\mathrm{CoA}$ in Patt1 structure. In the $\alpha 4$ helix there are two highly conserved phenylalanine residues present: Phe185 and Phe186 that are directed to CoA substrate. With the next highly conserved Phe192 residue located within $\alpha 4-\beta 6$ loop that follows $\alpha 4$ helix they form a system of stacking rings. CoA adenine rings are trapped in contacts with the plane of the Phe1 85 ring. These interactions contribute to proper positioning of the ligand. Structural alignment of Patt1 and RimI reveals that spatial localization of Phe186 residue in Patt1 corresponds to Tyr115 in RimI structure. Tyr115 (RimI) has been assigned a role of the active site acid [30]. The interactions with stacking rings systems have been shown to act as an alternative to general acid catalysis [31]. Therefore, it is proposed that this spatial arrangement of conserved phenolic rings should take part in acetyltransfer reaction (Fig. 4). In the structure of RimI the amide backbone of the residue Ile69 has a role of stabilizer of polarization of acetyl group in the process in which the tetrahedral intermediate is formed during acetyltransfer reaction. This position is occupied by a conserved Val140 residue in Patt1. In the structure of Patt1 in the
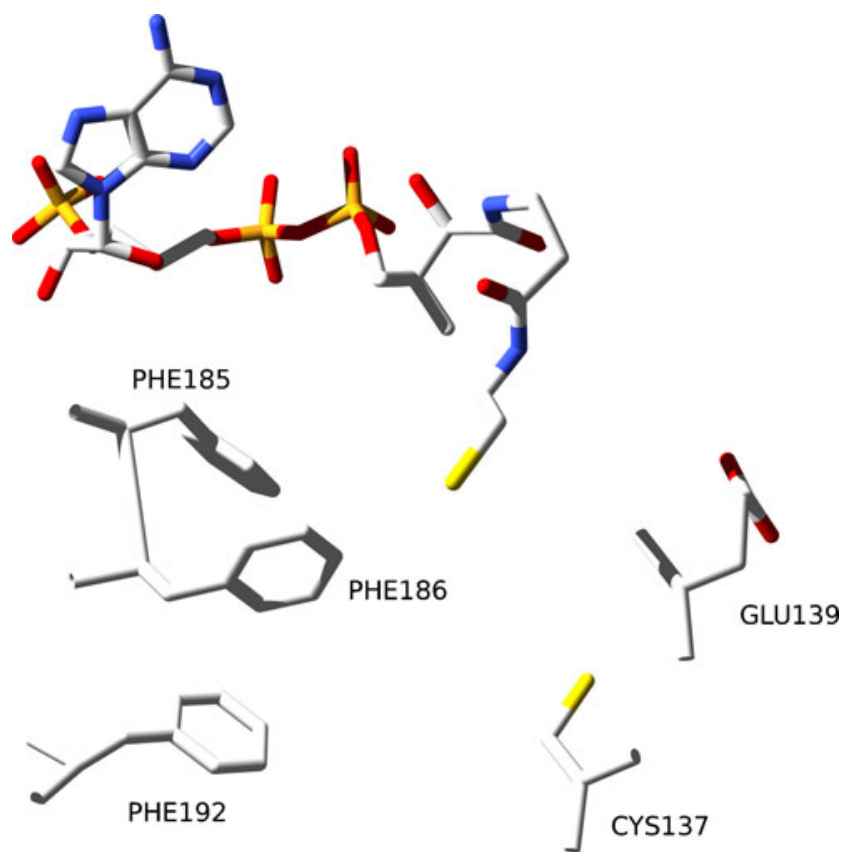

Fig. 4 Spatial localization of key residues in Patt1 active site. The CoA ligand was copied from RimI structure active site there is conserved Cysteine 137 residue located with sulfhydryl group directed to sulfhydryl group of CoA (Fig. 4). The presence of cysteine in the active site might suggest its involvement in reaction mechanism. Structural alignment of Patt1 model with yeast Esa1 structure [32] was performed. Esa1 is a histone acetyltransferase that belongs to the MYST subfamily. In Esa1 the strictly conserved Cys304 residue is responsible for the common acetylation reaction mechanism (that proceeds through an acetyl-cysteine enzyme intermediate) [33]. Both Patt1 and Esa1 perform acetylation of histone H4. The structural alignment reveals that both Patt1 Cys137 and Esa1 Cys304 residues are located in the active site, at the beginning of $\beta 4$ sheet. The Glu139 residue is localized in close proximity in the Patt1 model (within the $\beta 4$ sheet structural element) which has been assigned a role as an active site base [13]. Conserved residues are located in the region of specific interactions with the peptide that undergoes acetylation. These residues surround the peptide substrate and position it prior to acetyltransfer. Among them there are residues located in the $\alpha 1-\alpha 2$ loop. This loop is extended in comparison with RimI structure, due to probable insertion event. The most highly conserved residue in this regionTrp92 is engaged in stacking interactions with conserved Tyr85. This specific positioning increases the strength of hydrogen bonding interactions made by Tyr85 at the recognition interface of Patt1. The conserved Glu86 residue is directed into the space of peptide binding. In the loop that extends between $\beta 6-\beta 7$ sheet elements a sequence 'GCCG' (Gly203, Cys204, Cys205, Gly206) is located. Glycine residues facilitate conformational flexibility in this region. The presence of hydrophobic amino acid residue in the loop that is exposed to solvent indicates that the loop constitutes a part of recognition interface. In the structure of RimI in this region there are strictly conserved Tyr129 and Tyr130 residues that make contacts with the ligand peptide backbone [30]. Movements of these residues in RimI structure accompanies critical steps of acetyltransfer reaction. Met81(Patt1) is at the place of His23(RimI). Its proposed function, as in the case of RimI analog is to increase affinity to $\mathrm{CoA}$ by hydrophobic interactions.

\section{Conclusions}

The all-atom tertiary structure of a novel human histone acetyltransferase Patt1 was modeled by means of theoretical methods. The model was assessed with the state-of-the-art methods for protein structure validation, and subjected to 12 ns molecular dynamics simulation. The integrity of structure was retained. The model allowed to infer sequencestructure-function relationships of Patt1. The key residues identified as involved in acetyltransefer reaction were: Phe185, Phe186, Phe192, and Cys137. Patt1 promotes 
apoptosis in human hepatocellular carcinoma cell lines. The availability of effective therapy is strongly dependent on structural data of proteins. Three-dimensional molecular structure can be used as a map in performing protein design experiments and evaluation of their results. It also opens the possibility of rational drug design and can help in the development of personalized medicine.

Acknowledgments All calculations that required the use of high performance computing resources were carried out at the Academic Computer Center in Gdańsk.

Open Access This article is distributed under the terms of the Creative Commons Attribution License which permits any use, distribution, and reproduction in any medium, provided the original author(s) and the source are credited.

\section{References}

1. Loidl P (2001) Introduction: assembly, remodeling and modification of chromatin. CMLS Cell Mol Life Sci 58:663-664

2. Wu J, Grunstein M (2000) 25 years after the nucleosome model: chromatin modifications. Trends Biochem Sci 25(12):619-623

3. Strahl BD, Allis CD (2000) The language of covalent histone modifications. Nature 403(6765):41-45

4. Polevoda B, Sherman F (2002) The diversity of acetylated proteins. Genome Biol 3(5):reviews0006

5. Marmorstein R (2001) Structure of histone acetyltransferases. J Mol Biol 311:433-444

6. Marmorstein R (2001) Structure and function of histone acetyltransferases. CMLS Cell Mol Life Sci 58:693-703

7. Timmermann S, Lehrmann H, Polesskaya A, Harel-Bellan A (2001) Histone acetylation and disease. CMLS Cell Mol Life Sci 58:728-736

8. Di Gennaro E, Bruzzese F, Caraglia M, Abruzzese A, Budillon A (2004) Acetylation of proteins as novel target for antitumor therapy: review article. Amino Acids 26:435-441

9. Dyda F, Klein DC, Hickman AB (2000) GCN5-related Nacetyltransferases: a structural overview. Annu Rev Biophys Biomol Struct 29:81-103

10. Vetting MW, de Carvalho LPS, Yu M, Hedge SS, Magnet S, Roderick SL, Blanchard JS (2005) Structure and functions of the GNAT superfamily of acetyltransferases. Arch Biochem Biophys 433(1):212-226

11. Neuwald AF, Landsman D (1997) GCN5-related histone Nacetyltransferases belong to a diverse superfamily that includes the yeast SPT10 protein. Trends Biochem Sci 22(5):154-155

12. Brownell JE, Zhou J, Ranalli T, Kobayashi R, Edmondson DG, Roth SY, Allis CD (1996) Tetrahymena histone acetyltransferase A: a homolog to yeast Gcn5p linking histone acetylation to gene activation. Cell 84(6):843-851

13. Liu Z, Liu Y, Wang H, Ge X, Jin Q, Ding G, Hu Y, Zhou B, Chen Z, Ge X, Zhang B, Man X, Zhai Q (2009) Patt1, a novel protein acetyltransferase that is highly expressed in liver and downregulated in hepatocellular carcinoma, enchances apoptosis of hepatoma cells. Int J Biochem Cell Biol 41(12):2528-2537

14. Wheeler DL, Barret T, Benson DA, Bryant SH, Canese K, Chetvernin V, Church DM, Dicuccio M, Edgar R, Federhen S, Feolo M, Geer LY, Helmberg W, Kapustin Y, Khovayko O, Landsman D, Lipman DJ, Madden TL, Maglott DR, Miller V,
Ostell J, Pruitt KD, Schuler GD, Schumway M, Sequeira E, Sherry ST, Sirotkin K, Souvorov A, Starchenko G, Tatusov RL, Tatusova TA, Wagner L, Yashenko E (2008) Database resources of the National Center for Biotechnology information. Nucleic Acids Res 36(Database issue):D13-D21

15. Altschul SF, Madden TL, Schäffer AA, Zhang J, Zhang Z, Miller W, Lipman DJ (1997) Gapped BLAST and PSI-BLAST: a new generation of protein database search programs. Nucleic Acids Res 25(17): 3389-3402

16. Edgar RC (2004) MUSCLE: multiple sequence alignment with high accuracy and high throughput. Nucleic Acids Res 32(5):1792-1797, Print 2004

17. Edgar RC (2004) MUSCLE: a multiple sequence alignment method with reduced time and space complexity. BMC Bioinforma 5:113

18. Marchler-Bauer A, Bryant SH (2004) CD-search: protein domain annotations on the fly. Nucleic Acids Res 32(Web Server issue): W327-W331

19. Hall TA (1999) BioEdit: a user-friendly biological sequence alignment editor and analysis program for Windows 95/98/NT. Nucleic Acids Symp Ser 41:95-98

20. Galtier N, Gouy M, Gautier C (1996) SEAVIEW and PHYLO WIN: two graphic tools for sequence alignment and molecular phylogeny. Comput Appl Biosci 12(6):543-548

21. Thompson JD, Gibson TJ, Plewniak F, Jeanmougin F, Higgins DG (1997) The CLUSTAL_X windows interface: flexible strategies for multiple sequence alignment aided by quality analysis tools. Nucleic Acids Res 25(24):4876-4882

22. Page RD (1996) TreeView: an application to display phylogenetic trees on personal computers. Comput Appl Biosci 12(4): $357-358$

23. Kurowski MA, Bujnicki JM (2003) GeneSilico protein structure prediction meta-server. Nucleic Acids Res 31(13):38043807

24. Sali A, Blundell TL (1993) Comparative protein modelling by satisfaction of spatial restraints. J Mol Biol 234(3):779-815

25. Guex N, Peitsch MC (1997) SWISS-MODEL and the Swiss-Pdb viewer: an environment for comparative protein modeling. Electrophoresis 18(15):2714-2723

26. Pawłowski M, Gajda JM, Matlak R, Bujnicki JM (2008) MetaMQAP: a meta-server for the quality assessment of protein models. BMC Bioinforma 9:403

27. Wallner B, Elofsson A (2006) Identification of correct regions in protein models using structural, alignment, and consensus information. Protein Sci 15(4):900-913

28. Wallner B, Elofsson A (2003) Can correct protein models be identified? Protein Sci 12(5):1073-1086

29. Case DA, Cheatham TE 3rd, Darden T, Gohlke H, Luo R, Merz KM Jr, Onufriev A, Simmerling C, Wang B, Woods RJ (2005) The Amber biomolecular simulation programs. J Comput Chem 26(16):16681688

30. Vetting MW, Bareich DC, Yu M, Blanchard JS (2008) Crystal structure of RimI from Salmonella typhimurium LT2, the GNAT responsible for N(alpha)-acetylation of ribosomal protein S18. Protein Sci 17(10):1781-1790

31. Versées W, Loverix S, Vandemeulebroucke A, Geerlings P, Steyaert J (2004) Leaving group activation by aromatic stacking: an alternative to general acid catalysis. J Mol Biol 338(1):1-6

32. Yan Y, Barlev NA, Haley RH, Berger SL, Marmorstein R (2000) Crystal structure of yeast Esal suggests a unified mechanism for catalysis and substrate binding by histone acetyltransferases. Mol Cell 6(5):1195-1205

33. Yan Y, Harper S, Speicher DW, Marmorstein R (2002) The catalytic mechanism of the ESA1 histone acetyltransferase involves a selfacetylated intermediate. Nat Struct Biol 9(11):862-869 